\title{
РЕШЕНИЕ ПРОБЛЕМЫ СОБСТВЕННЫХ ЗНАЧЕНИЙ ОПЕРАТОРА ЛАПЛАСА В ПРОСТРАНСТВЕ СОЛЕНОИДАЛЬНЫХ В ДВУМЕРНОЙ ПРЯМОУГОЛЬНОЙ ОБЛАСТИ ФУНКЦИЙ, ОДНОРОДНЫХ НА ЧАСТИ ГРАНИЦЫ
}

\author{
(Представил Я. Эйнасто)
}

Настоящая статья является продолжением [ $\left.{ }^{1}\right]$. При ссылке на формулы работы [ $\left.{ }^{1}\right]$ будем использовать обозначения $(1.1),(1.2)$ и т. д.

\section{1. Формулировка задачи}

Рассмотрим проблему собственных значений оператора Лапласа $\Delta=$ $=\partial^{2} /\left(\partial x^{1}\right)^{2}+\partial^{2} /\left(\partial x^{2}\right)^{2}$ в области (1.1). В классической постановке задача выглядит так:

найти собственные функции $\mathbf{u}^{(k)}, \varphi^{(k)}$ и собственные числа $\lambda^{(k)}$ уравнений

$$
\begin{gathered}
\Delta \mathbf{u}+\lambda \mathbf{u}=\operatorname{grad} \varphi, \\
\operatorname{div} \mathbf{u}=0
\end{gathered}
$$

при дополнительных условиях

$$
\begin{gathered}
\left.\mathbf{u}\right|_{x^{2}=0}=0,\left.\quad \mathbf{u}\right|_{x^{2}=1}=0, \\
\left.\mathbf{u}\right|_{x^{4}=0}=\left.\mathbf{u}\right|_{x^{4}=a^{-1}},\left.\quad \varphi\right|_{x^{4}=0}=\left.\varphi\right|_{x^{4}=a^{-1}} .
\end{gathered}
$$

Эта задача решается в слабой (вариационной) постановке. Кроме того, изучаются основные свойства решения, в частности полнота системы $\left\{\mathbf{u}^{(k)}\right\}$ в пространствах $U^{q}(1.15)$.

Система собственных функций $\left\{\mathbf{u}^{(k)}, \varphi^{(k)}\right\}$ имеет многочисленные приложения в гидродинамике. С ее помощью полностью описывается периодическое в направлении $x^{1}$ решение нестационарного уравнения НавьеСтокса при нулевом числе Рейнольдса и может быть исследована устойчивость стационарного решения. Проектирование уравнений НавьеСтокса в систему $\left\{\mathbf{u}^{(k)}\right\}$ дает простые и удобные алгоритмы для численного моделирования двумерных турбулентных течений в бесконечных каналах $\left[{ }^{2}\right]$. Весьма существенный вклад решения задачи (1) и (2) могут внести также в исследование аттракторного механизма турбулентности, существование которого докаказно в [ $\left.{ }^{3}\right]$ (см. также $\left.[4,5]\right)$.

\section{2. Вариационная формулировка}

Умножением (1) на произвольный вектор $\overline{\mathbf{v}} \in U^{1}$ и интегрированием по частям получим вариационное уравнение

$$
\sum_{\alpha=1}^{2} \int_{\Omega} \mathbf{u}, \alpha \overline{\mathbf{v}}_{, \alpha} \mathrm{d} x=\lambda \int_{\Omega} \mathbf{u} \overline{\mathbf{v}} \mathrm{d} x, \quad \mathrm{~V} \mathbf{v} \in U^{1},
$$

где $\mathbf{u}_{, \alpha}=\partial \mathbf{u} / \partial x^{\alpha}$, или в более компактной записи 


$$
(\mathbf{u}, \alpha, \mathbf{v}, \alpha)=\lambda(\mathbf{u}, \mathbf{v}), \quad \forall \mathbf{v} \in U^{1} .
$$

3 а м е ани е 1. Требование $\mathbf{v} \in U^{1}$ является необходимым. В менее гладких пространствах может не существовать $\mathbf{v}, \alpha$.

3 а м е ч н и е 2. В (3) не входит неизвестная функция $\varphi$. Это следствие того, что

$$
\int_{\Omega} \operatorname{grad} \varphi \overline{\mathbf{v}} \mathrm{d} x=\int_{\Omega} \operatorname{div}(\varphi \overline{\mathbf{v}}) \mathrm{d} x-\int_{\Omega} \varphi \operatorname{div} \overline{\mathbf{v}} \mathrm{d} x=0,
$$

если только $\mathbf{v} \in U^{1}$ и $\left.\varphi\right|_{x^{1}=0}=\left.\varphi\right|_{x^{1}=a^{-1}}$. Следовательно, в вариационной постановке задача упрощается; нужно найти неизвестную функцию u и число $\lambda$. Функцию же $\varphi$ можно определить при известных и и $\lambda$ из уравнений (1).

Из $\mathbf{v} \in U^{\prime}$ следует $\mathbf{v}, \mathbf{v}_{, \alpha} \in L_{2}(\Omega)$, поэтому из вариационного уравнения (3) вытекает включение $\mathbf{u}, \mathrm{u}_{, \alpha} \in L_{2}(\Omega)$. Вместе с условиями (2) это означает, что $\mathbf{u} \in U^{1}$. Разложим $\mathbf{u}$ и $\mathbf{v}$ с учетом $U^{1} \subset S^{1}$ в базисе $\left\{\mathbf{s}_{m n}\right\}$ $(1.10)$

$$
\begin{array}{ll}
\mathbf{u}=\sum_{m, n} u_{m n} \mathbf{s}_{m n}, & \mathbf{v}=\sum_{m, n} v_{m n} \mathbf{s}_{m n}, \\
u_{m n}=\left(\mathbf{u}, \mathbf{s}_{m n}\right), & v_{m n}=\left(\mathbf{v}, \mathbf{s}_{m n}\right)
\end{array}
$$

(как следует из (1.15), коэффициенты при $\mathrm{s}^{0}$ можно положить равными нулю). Тогда (3) примет вид

$$
\begin{gathered}
\sum_{m, n} \mathbf{k}_{m n}^{2} u_{m n} \bar{v}_{m n}=\lambda \sum_{m, n} u_{m n} \bar{v}_{m n}, \\
\sum_{n} \alpha_{m n} u_{m n}=\sum_{n} \beta_{m n} u_{m n}=0 \quad \forall m, \\
\mathrm{Vv}: \sum_{n} \alpha_{m n} v_{m n}=\sum_{n} \beta_{m n} v_{m n}=0 \quad \forall m,
\end{gathered}
$$

где (6) выражает условие $\mathbf{u} \in U^{1}$, а (7) означает, что уравнение (5) должно выполняться при пронзвольных векторах $\mathbf{v} \in U^{1}$.

Устанавливаем некоторые свойства решения системы (5)-(7).

1. Собственные числа $\lambda$ действительные и неотрицательные. Чтобы в этом убедиться, достаточно положить $\mathbf{v}=\mathbf{u}$ в уравнениях (5).

2. Так как $\lambda, \underset{\mathrm{k}_{n}}{\mathbf{2}}, \boldsymbol{\alpha}_{m n}, \beta_{m n}-$ действительные числа, то $\left\{u_{m n}\right\}-$ действительный вектор (но это не означает, что и (см. (4)) будет действительной функцией). Соответственно в дальнейшем в (5)-(7) можно ограничиваться действительными $\bar{v}_{m n}=v_{m n}$.

3. Из симметричности уравнения (5) (при $u_{m n}=\bar{u}_{m n}, v_{m n}=\bar{v}_{m n}$ ) относительно $\mathbf{v} \leftrightarrow \mathbf{u}$ следует ортогональность собственных векторов, отвечающих различным собственным числам, в $L_{2}(\Omega)$

$$
\left(\mathbf{u}^{(1)}, \mathbf{u}^{(2)}\right)=\sum_{m, n} u_{m n}^{(1)} u_{m n}^{(2)}=0, \quad \lambda^{(1)} \neq \lambda^{(2)} .
$$

Кроме того, если выполнено условие ортогональности (8), то из (5) следует $\left(\mathbf{u}_{, \alpha^{(1)}}, \mathbf{u}_{, \alpha^{(2)}}\right)=0$, поэтому

$$
\left(\mathbf{u}^{(1)}, \mathbf{u}^{(2)}\right)_{W_{2}^{\prime}(\Omega)}=\left(\mathbf{u}^{(1)}, \mathbf{u}^{(2)}\right) \mathrm{s}^{\mathbf{1}}=0 .
$$

\section{3. Решение в ортогональном базисе}

Варьирование (5) по $\bar{v}_{m n}$ с учетом (7) приводит к уравнениям 


$$
\left(\mathbf{k}_{m n}^{2}-\lambda\right) u_{m n}=p_{m} \alpha_{m n}+q_{m} \beta_{m n},
$$

где множители Лагранжа $p_{m}, q_{m}$ следует определить из условий (6).

3 а м е ч а и е. Так как $\left\{\alpha_{m n}\right\}_{n},\left\{\beta_{m n}\right\}_{n}$ определяют не векторы, а непрерывные линейные функционалы в $U^{1}$, то (10) представляет собой функциональное уравнение в сопряженном пространстве $\left(U^{1}\right)^{\prime}$.

С учетом действительности собственных чисел $\lambda$ могут встречаться два случая:

1) $\mathbf{k}_{m n}^{2} \neq \lambda$

ни при каких $m, n$,

2) $\mathbf{k}_{m_{0} n_{0}}^{2}=\lambda$.

В первом случае получим из (10)

$$
u_{m n}=\left(p_{m} \alpha_{m n}+q_{m} \beta_{m n}\right) /\left(\mathbf{k}_{m n}^{2}-\lambda\right) .
$$

Чтобы определить $p_{m}$ и $q_{m}$, подставим это решение в $(6)$ :

$$
\begin{array}{ll}
p_{m} \sum_{n} \alpha_{m n}^{2} /\left(\mathbf{k}_{m n}^{2}-\lambda\right)+q_{m} \sum_{n} \alpha_{m n} \beta m n /\left(\mathbf{k}_{m n}^{2}-\lambda\right)=0 & \forall m, \\
p_{m} \sum_{n} \alpha_{m n} \beta m n /\left(\mathbf{k}_{m n}^{2}-\lambda\right)+q_{m} \sum_{n} \beta_{m n}^{2} /\left(\mathbf{k}_{m n}^{2}-\lambda\right)=0 & \forall m .
\end{array}
$$

Ho

$$
\sum_{n} \alpha_{m n} \beta_{m n} /\left(\mathbf{k}_{m n}^{2}-\lambda\right)=0 \quad \forall m
$$

поэтому, введя характеристические функции

$$
\begin{aligned}
& S_{m}(\lambda)=\sum_{n} \alpha_{m n}^{2} /\left(\mathbf{k}_{m n}^{2}-\lambda\right), \\
& T_{m}(\lambda)=\sum_{n} \beta_{m n}^{2} /\left(\mathbf{k}_{m n}^{2}-\lambda\right),
\end{aligned}
$$

получим следующие основные решения

a)

$$
\begin{aligned}
& p_{m}=1, \quad S_{m}(\lambda)=0 ; \\
& p_{m^{\prime}}=0, m^{\prime} \neq m ; q_{m}=0 \\
& u_{m^{\prime} n}= \begin{cases}\alpha_{m n} /\left(\mathbf{k}_{m n}^{2}-\lambda\right), & m^{\prime}=m, \\
0, & m^{\prime} \neq m ;\end{cases}
\end{aligned}
$$

б)

$$
\begin{aligned}
& q_{m}=1, \quad T_{m}(\lambda)=0 ; \\
& q_{m^{\prime}}=0, m^{\prime} \neq m ; p_{m}=0 \\
& u_{m^{\prime} n}= \begin{cases}\beta_{m n} /\left(\mathbf{k}_{m n}^{2}-\lambda\right), & m^{\prime}=m, \\
0, & m^{\prime} \neq m .\end{cases}
\end{aligned}
$$

Легко увидеть, что все другие решения (которые имеются, если, например, $S_{m}(\lambda)=S_{m_{1}}(\lambda)=0$ или $S_{m}(\lambda)=T_{m_{1}}(\lambda)=0$ и т. д.), являются линейными комбинациями векторов (12) и (13). Итак, (12) и (13) полностью исчерпывают случай 1).

Исследование случая 2) (которое представляется элементарным, но весьма громоздким занятием) показывает, что нетривиальные решения существуют при $m_{0}=0, n_{0} \neq 0$ и имеют вид 


$$
\begin{aligned}
& u_{m n}=0, m \neq 0 \\
& u_{0 n}= \begin{cases}1, & n= \pm n_{0} \neq 0 \\
0, & n \neq \pm n_{0}\end{cases}
\end{aligned}
$$

при этом $\lambda^{\left(n_{0}\right)}=\mathbf{k}^{2}{ }_{0 n_{0}}=4 \pi^{2} n_{0}^{2}$.

\section{4. Характеристические уравнения}

В случае решений (14) нам известны собственные числа $\lambda$. Чтобы определить собственные числа, отвечающие векторам (12) и (13), нужно решить характеристические уравнения

$$
S_{m}(\lambda)=0, \quad T_{m}\left(\lambda^{\prime}\right)=0 .
$$

С учетом явных выражений для $\alpha_{m n}$ и $\beta_{m n}$ (см. (1.5)) характеристические уравнения примут следующий вид:

$$
\begin{aligned}
& S_{m}(\lambda)=\left(1 / 4 \pi^{2}\right) \sum_{n=-\infty}^{\infty} n^{2}\left(n^{2}+a^{2} m^{2}\right)^{-1}\left(n^{2}+a^{2} m^{2}-\lambda / 4 \pi^{2}\right)^{-1} \\
& T_{m}(\lambda)=\left(a^{2} m^{2} / 4 \pi^{2}\right) \sum_{n=-\infty}^{\infty}\left(n^{2}+a^{2} m^{2}\right)^{-1}\left(n^{2}+a^{2} m^{2}-\lambda / 4 \pi^{2}\right)^{-1}
\end{aligned}
$$

(при $m=0$ имеем $T_{0}(\lambda) \equiv 0$ и (13) не представляет в этом случае решения) .

Отсюда вытекают дальнейшие свойства решений.

4. При фиксированном $m$ характеристические числа $\lambda$, отвечающие собственным функциям (12) и (13), удовлетворяют неравенству

$$
\lambda>4 \pi^{2} a^{2} m^{2} .
$$

5. Так как $S_{m}(\lambda)=S_{-m}(\lambda), T_{m}(\lambda)=T_{-m}(\lambda)$, то каждому собственному числу $\lambda-$ решению уравнения $S_{m}(\lambda)=0$ или $T_{m}(\lambda)=0$ - отвечают по крайней мере два вектора (12) или (13) с отличными от нуля компонентами $u_{m^{\prime} n}$ при $m^{\prime}=m$ и $m^{\prime}=-m$ соответственно.

Разлагая ряды (15) по $n$ на простейшие дроби и используя формулы суммирования :[ $\left.{ }^{6}\right]$

$$
\sum_{n=-\infty}^{\infty}\left(n^{2}+\alpha^{2}\right)^{-1}=\pi \operatorname{cth}(\pi \alpha) / \alpha, \quad \sum_{n=-\infty}^{\infty}\left(n^{2}-\alpha^{2}\right)^{-1}=\pi \operatorname{ctg}(\pi \alpha) / \alpha,
$$

характеристические уравнения можно привести к виду (учитывая при этом (16))

$S_{m}(\lambda)=(\pi / \lambda)\left[\operatorname{am} \operatorname{cth}(\pi a m)-\sqrt{\lambda / 4 \pi^{2}-a^{2} m^{2}} \operatorname{ctg}\left(\pi \sqrt{\lambda / 4 \pi^{2}-a^{2} m^{2}}\right)\right]$,

$$
\begin{aligned}
& T_{m}(\lambda)=-\left(\pi^{2} a^{2} m^{2} / \lambda\right)[\operatorname{cth}(\pi a m) / a m+ \\
& \left.+\operatorname{ctg}\left(\pi \sqrt{\lambda / 4 \pi^{2}-a^{2} m^{2}}\right) / \sqrt{\lambda / 4 \pi^{2}-a^{2} m^{2}}\right] .
\end{aligned}
$$

Уравнение $S_{0}(\lambda)=0$, как следует йз (17), имеет корни

$$
\lambda_{0 j}=4 \pi^{2}(j+1 / 2)^{2}, \quad j=0,1, \ldots .
$$

Уравнение $S_{m}(\lambda)=0$ с заменой $\lambda \rightarrow \mu$

$$
\lambda=4 \pi^{2}\left(\mu^{2}+a^{2} m^{2}\right)
$$

приводится к виду 


$$
\operatorname{ctg}(\pi \mu)=\operatorname{am} \operatorname{cth}(\pi a m) / \mu
$$

и имеет простые корни $\mu_{m j}$

$$
1 \leqslant j<\mu_{m j}<j+1 / 2 .
$$

Наконец, уравнение $T_{m}(\lambda)=0$ заменой переменного $\lambda \rightarrow v$

$$
\lambda=4 \pi^{2}\left(v^{2}+a^{2} m^{2}\right)
$$

приводится к виду

$$
\operatorname{ctg}(\pi v)=-v \operatorname{cth}(\pi a m) / a m
$$

и имеет простые корни $v_{m j}$

$$
0<j-1 / 2<v_{m j}<j
$$

\section{5. Решение в координатном представлении}

Итак, собственные функции в базисе $\left\{\mathbf{s}_{m n}\right\}$ определяются формулами (12) - (14). Эти же функции в координатном представлении будут иметь вид

$$
\begin{gathered}
\mathbf{u}_{0 j}^{1}(\mathbf{x})=\mathbf{s}_{0 j}+\mathbf{s}_{0,-j}=\mathbf{e}^{1} i 2 \sqrt{a} \sin \left(2 \pi j x^{2}\right), \\
\lambda_{0 j}^{(1)}=4 \pi^{2} j^{2}, \quad j=1,2, \ldots ; \\
\mathbf{u}_{0 j}^{2}(\mathbf{x})=\sum_{n=-\infty}^{\infty} \mathbf{s}_{0 n}(\mathbf{x}) \alpha_{0 n} /\left(\mathbf{k}_{0 n}^{2}-\lambda_{0 j}^{(2)}\right), \\
\lambda_{0 j}^{(2)}=4 \pi^{2}(j+1 / 2)^{2}, \quad j=0,1, \ldots ; \\
\mathbf{u}_{m j}^{1}(\mathbf{x})=\sum_{n=-\infty}^{\infty} \mathbf{s}_{m n}(\mathbf{x}) \alpha_{m n} /\left(\mathbf{k}_{m n}^{2}-\lambda_{m j}^{(1)}\right), \\
\lambda_{m j}^{(1)}=4 \pi^{2}\left(\mu_{m j}^{2}+a^{2} m^{2}\right), \quad j=1,2, \ldots ; \\
\mathbf{u}_{m j}^{2}(\mathbf{x})=\sum_{n=-\infty}^{\infty} \mathbf{s}_{m n}(\mathbf{x}) \beta_{m n} /\left(\mathbf{k}_{m n}^{2}-\lambda_{m j}^{(2)}\right), \\
\lambda_{m j}^{(2)}=4 \pi^{2}\left(v_{m j}^{2}+a^{2} m^{2}\right), \quad j=1,2, \ldots ;
\end{gathered}
$$

где $\mu_{m j}, v_{m j}-$ решения уравнений (22) и (25).

Отсюда получим следующие свойства решения.

6. Система (27) является ортогональной в $L_{2}(\Omega)$. При различных $m$ взаимная ортогональность обусловлена тем, что функции принадлежат различным ортогональным подпространствам. Ортогональность $\left(\mathbf{u}^{1}{ }_{m j}, \mathbf{u}^{2}{ }_{m j}\right)=0$ обусловлена тем, что $\alpha_{m n}=-\alpha_{m,-n}, \boldsymbol{\beta}_{m n}=\beta_{m,-n}$. Ортогональность же $\left(\mathbf{u}^{1}{ }_{0 j}, \mathbf{u}^{2}{ }_{0 j^{\prime}}\right)=0,\left(\mathbf{u}^{1}{ }_{m j}, \mathbf{u}^{1}{ }_{m j^{\prime}}\right)=0,\left(\mathbf{u}^{2}{ }_{m j}, \mathbf{u}^{2}{ }_{m j^{\prime}}\right)=0$ следует из свойства 1 , если учесть, что соответствующие собственные числа являются простыми и не совпадают.

7. Система (27) ортогональна также в $S^{1}\left(\right.$ и в $\left.W_{2}^{1}(\Omega)\right)$. Это следствие свойств 3 и 6.

8. Система (27) принадлежит пространствам $S^{q}$ при $q<3 / 2$. Действительно,

$$
\left\|\mathbf{u}_{0 j}^{1}\right\|_{S^{q}}^{2}=2\left(1+\mathbf{k}_{0 j}^{2}\right)^{q},
$$




$$
\begin{aligned}
& \left\|\mathbf{u}_{0 j}^{2}\right\|_{S^{q}}^{2}=\left(1 / 4 \pi^{2}\right)^{2} \sum_{n}\left(1+\mathbf{k}_{0 n}^{2}\right)^{q} /\left[n^{2}-(j+1 / 2)^{2}\right]^{2}, \\
& \left\|\mathbf{u}_{m j}^{1}\right\|_{S^{q}}^{2}<\left(1 / 4 \pi^{2}\right)^{2} \sum_{n}\left(1+\mathbf{k}_{m n}^{2}\right)^{q} /\left(n^{2}-\mu_{m j}^{2}\right)^{2}, \\
& \left\|\mathbf{u}_{m j}^{2}\right\|_{S^{q}}^{2}<\left(1 / 4 \pi^{2}\right)^{2} \sum_{n}\left(1+\mathbf{k}_{m n}^{2}\right)^{q} /\left(n^{2}-v_{m j}^{2}\right)^{2} .
\end{aligned}
$$

Ряды сходятся при $q<3 / 2$, так как при больших $n$

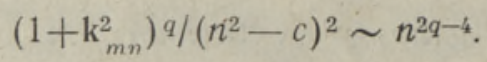

9. Собственные функции принадлежат пространствам $U q, 1 / 2<q<3 / 2$. Это вытекает из предыдущего свойства, если учесть, что собственные векторы ортогональны (см. (6)) функционалам $a_{m}$ и $b_{m}(1.12)$.

Ряды (27) допускают явное суммирование. Для этого нужно $\alpha_{m n}, \beta_{m n}$ и $\mathbf{S}_{m n}$ заменить их явными выражениями (1.4)-(1.5), разложить получающиеся суммы на простейшие дроби по $n$ и использовать формулы суммирования $\left[{ }^{6}\right]$ '

$$
\begin{gathered}
\sum_{n=1}^{\infty} \cos (2 \pi y n) /\left(n^{2}+\alpha^{2}\right)=-1 /\left(2 \alpha^{2}\right)+\pi \operatorname{ch},[\pi \alpha(1-2 y)] /[2 \alpha \operatorname{sh}(\pi \alpha)], \\
\sum_{n=1}^{\infty} n \sin (2 \pi y n) /\left(n^{2}+\alpha^{2}\right)=\pi \operatorname{sh}[\pi \alpha(1-2 y)] /[2 \operatorname{sh}(\pi \alpha)],
\end{gathered}
$$

а также формулы, вытекающие отсюда при замене $\alpha \rightarrow i \alpha$. Если-опустить несущественные множители, то результат суммирования таков:

$$
\begin{aligned}
& \mathbf{u}_{0 j}^{1}=\mathrm{e}^{1} \sin \left(2 \pi j x^{2}\right), \quad \mathbf{u}_{0 j}^{2}=\mathbf{e}^{1} \sin \left[\pi(2 j+1) x^{2}\right], \\
& \mathbf{u}_{m j}^{1}=\exp \left(i 2 \pi a m x^{1}\right)\left[\mathbf{e}^{1} p_{m}\left(x^{2}, \mu_{m j}\right)-\mathbf{e}^{2} i a m q_{m}\left(x^{2}, \mu_{m j}\right)\right], \\
& \mathbf{u}_{m j}^{2}=\exp \left(i 2 \pi a m x^{1}\right)\left[\mathbf{e}^{1} q_{m}\left(x^{2}, v_{m j}\right)-\mathbf{e}^{2} i a m r_{m}\left(x^{2}, v_{m j}\right)\right],
\end{aligned}
$$

где

$p_{m}(y, \zeta)=a m \operatorname{ch}[\pi a m(1-2 y)] / \operatorname{sh}(\pi a m)-\zeta \cos [\pi \zeta(1-2 y)] / \sin (\pi \zeta)$,

$q_{m}(y, \zeta)=-\operatorname{sh}[\pi a m(1-2 y)] / \operatorname{sh}(\pi a m)+\sin [\pi \zeta(1-2 y)] / \sin (\pi \zeta)$,

$r_{m}(y, \zeta)=\operatorname{ch}[\pi a m(1-2 y)] /[\operatorname{am} \operatorname{sh}(\pi a m)]+\cos [\pi \zeta(1-2 y)] /[\zeta \sin (\pi \zeta)]$

$\left(\mathrm{u}_{0 j}{ }^{1}, \mathrm{u}_{0 j}{ }^{2}\right.$, очевидно, образуют единую систему функций вида $\left.\mathrm{e}^{1} \sin \left(\pi j x^{2}\right), j=1,2, \ldots\right)$. На основании этих формул устанавливаются следующие свойства собственных векторов.

10. Собственные векторы являются бесконечно дифференцируемыми периодическими соленоидальными функциями, обращающимися в 0 в каждой точке $x^{2}=0, x^{2}=1,0 \leqslant x^{1} \leqslant a^{-1}$ н, следовательно, принадлежащими множеству $\mathfrak{i}^{1}$ (1.35) (но не принадлежащими $\mathcal{U}^{p}, p \geqslant 2$, так как их производные уже не являются периодическими функциями).

11. Собственные векторы удовлетворяют «классическому» уравнению (1)

где

$$
\begin{gathered}
\Delta \mathbf{u}_{0 j}^{\alpha}+\lambda_{0 j}^{(\alpha)} \mathbf{u}_{0 j}^{\alpha}=0, \\
\Delta \mathbf{u}_{m j}^{\alpha}+\lambda_{m j}^{(\alpha)} \mathbf{u}_{m j}^{\alpha}=\operatorname{grad} \varphi_{m j}^{\alpha},
\end{gathered}
$$

$$
\begin{aligned}
\varphi_{m j}^{1} & =-\left[i \lambda_{m j}^{(1)} / 2 \pi \operatorname{sh}(\pi a m)\right] \exp \left(i 2 \pi a m x^{1}\right) \operatorname{ch}\left[\pi a m\left(1-2 x^{2}\right)\right], \\
\varphi_{m j}^{2} & =-\left[i \lambda_{m j}^{(2)} / 2 \pi a m \operatorname{sh}(\pi a m)\right] \exp \left(i 2 \pi a m x^{1}\right) \operatorname{sh}\left[\pi a m\left(1-2 x^{2}\right)\right] .
\end{aligned}
$$




\section{6. Полнота системы собственных векторов}

Решение задачи (5)-(7) обладает свойством

12. Система (29) полна в пространствах $U q$ при $1 / 2<q<3 / 2$. Если $q=1$, то утверждение можно доказать, используя следующий результат о компактности:

Л е м м а. Множество $D$, определяемое уравнениями

$$
\begin{aligned}
& u \in D: \sum_{m, n} \mathrm{k}_{m n}^{2} u_{m n} \overline{u_{m n}}=1, \\
& \sum_{n} \alpha_{m n} u_{m n}=\sum_{n} \beta_{m n} u_{m n}=0 \quad \mathrm{~V} m,
\end{aligned}
$$

компактно в Sò. $^{\text {. }}$

Наметим доказательство в общих чертах при произвольном $q$ : $1 / 2<q<3 / 2$.

Решаем уравнения $(5)-(7)$ в конечномерном пространстве $S(M, N)$ (1.17). Легко установить, что решения $u^{j}=\left\{u^{j}{ }_{m n}\right\} \in S(M, N), j=1, \ldots$ $\ldots, \operatorname{dim} U(M, N)$ образуют базис в пространстве $U(M, N)($ см. (1.16)). При $M, N \rightarrow \infty$ эти решения стремятся в метрике $S^{q}$ к векторам (12)(14), если $1 / 2<q<3 / 2$. Отсюда на основании леммы 1 [ ${ }^{1}$ ] следует наше утверждение.

Из свойств 7 и 12 получим

13. Система собственных векторов (29)- образует базис в пространстве $U^{1}$.

До сих пор мы рассматривали пространства $U^{q}$ при $q>1 / 2$. Если доопределить $U^{q}$ при $q \leqslant 1 / 2$ соотношением

$$
U q=\left[U^{1}\right] s^{q}=\left[\mathcal{U}^{1}\right] s^{q},
$$

то, очевидно, справедливым будет следующее утверждение:

14. Система (29) является полной в пространствах $U^{q}$ при $q \leqslant 1 / 2$ и образует базис в $U^{0}$.

Особый интерес для гидродинамических приложений представляет пространство $U^{0}$. Имеем

$$
U^{0} \subset S^{0}
$$

(определение пространства соленоидальных векторов $S^{0}$ см. (1.10)). Это вложение не является плотным. В самом деле, система (29) построена так, что каждый вектор $\mathbf{u}_{m j}^{\alpha}$ ортогонален системе функционалов $\left\{\mathrm{a}_{m}, \mathbf{b}_{m}, \mathbf{1}^{0}\right\}$ (см. (1.12)), поэтому пространство $U^{0}$, являясь замыканием линейной оболочки системы (29) в $S^{0}$, совпадает с ядром функционалов $\left\{\mathbf{a}_{m}, \mathbf{b}_{m}, 1^{0}\right\}$ в $S^{0}$. Функционалы $\mathbf{a}_{m}$ не ограничены в $S^{0}$ ([ $\left.{ }^{1}\right]$, теорема 1$)$, поэтому их ядро всюду плотно в $S^{0}\left[{ }^{7}\right]$. Функционалы $\mathbf{b}_{m}, 1^{0}$ же ограничены в $S^{0}$ и выделяют в нем замкнутое подпространство, которое и есть $U^{0}$. Из ограниченности $\mathbf{b}_{m}$ и $1^{0}$ в пространстве $S^{0}$ следует существование векторов $\mathbf{b}_{m}{ }^{0}, \mathbf{s}^{0} \in S^{0}$, таких что $\left\langle\mathbf{b}_{m}, \mathbf{u}\right\rangle=\left(\mathbf{u}, \mathbf{b}_{m}{ }^{0}\right),\left\langle 1^{0}, \mathbf{u}\right\rangle=\left(\mathbf{u}, \mathbf{s}^{0}\right)$, $\forall \mathbf{u} \in \mathcal{S}^{0}$. Әти векторы имеют вид

$$
\begin{gathered}
\mathbf{b}_{m}^{0}=\sum_{n} \beta_{m n} \mathbf{s}_{m n}=a^{3 / 2} m \sum_{n} \exp \left(i \mathbf{k}_{m n} \mathbf{x}\right)\left(\mathbf{e}^{1} n-\mathbf{e}^{2} a m\right) /\left(a^{2} m^{2}+n^{2}\right)= \\
=\left[\pi a^{3 / 2} m / \operatorname{sh}(\pi a m)\right] \exp \left(i 2 \pi a m x^{1}\right)\left\{-i \mathbf{e}^{1} \operatorname{sh}\left[\pi a m\left(1-2 x^{2}\right)\right]+\right. \\
\left.+\mathbf{e}^{2} \operatorname{ch}\left[\pi a m\left(1-2 x^{2}\right)\right]\right\} .
\end{gathered}
$$

Они являются взаимно ортогональными, а также ортогональны всем векторам (29). Таким образом: 
15. Система (35) образует базис в ортогональном дополнении $W^{0}=$ $=S^{0} \backslash U^{0}$, а системы (29) и (35) образуют совместно базис в пространстве $S^{0}$.

Заметим также, что

$$
\begin{gathered}
\mathbf{s}^{0}=\operatorname{grad}\left(\sqrt{a} x^{2}\right), \\
\mathbf{b}_{m}^{0}=-[\sqrt{a} / \operatorname{sh}(\pi a m)] \operatorname{grad}\left\{\exp \left(i 2 \pi a m x^{1}\right) \operatorname{sh}\left[\pi a m\left(1-2 x^{2}\right)\right]\right\}, \\
\operatorname{div} s^{0}=0, \quad \operatorname{div} \mathbf{b}_{m}^{0}=0,
\end{gathered}
$$

поэтому $\mathbf{s}^{0}$ и $\mathbf{b}_{m}{ }^{0}$ являются в области $\Omega$ гармоническими функциями.

\section{7. Заключительные замечания}

Основные достоинства системы (29) заключаются в том, что она диагонализирует оператор Лапласа в пространствах $U^{0}, U^{1}$ и образует там ортогональный базис. Однако эта система не способна представить элементы пространства $U q$ при $q \geqslant 3 / 2$. Чтобы получить более гладкие базисные функции, следует использовать уравнение

$$
\sum_{m, n}\left(\mathbf{k}_{m n}^{2}\right)^{r} u_{m n} \overline{v_{m n}}=\lambda \sum_{m, n} u_{m n} \overline{v_{m n}} \quad \mathrm{~V} \mathbf{v} \subset U r
$$

при условиях (6), (7) и $r>1$. Так, например, при $r=2$ решения этой задачи образуют полную систему в $U^{q}$ при $q<7 / 2$.

Задача (1) при краевых условиях (2) приводит к вариационному уравнению (3) в пространстве $U^{1}$. Если вместо (2) рассмотреть краевое условие $\left.\mathbf{u}\right|_{r}=0$, то возникает вариационная задача в пространстве $V^{1}$ (1.20), которая может быть решена методами, развитыми в настоящей статье и в $\left[{ }^{7}\right]$. Отметим, что эта задача в случае произвольной области $\Omega$ изучена в $\left[{ }^{8,9}\right]$.

Другое направление, в котором полученные результаты допускают обобщение, возникает при выделении в $S^{q}$ (вместо $S^{q}$ можно взять'также $H^{q}$ или $G^{q}$ ) подпространств, отличных от $U^{q}$ или $V^{q}$. Напомним, что к пространствам $U^{q}$ мы пришли, рассматривая в $S^{q}$ функции, однородные на $\Gamma_{1}$. Но вместо однородности самих функций можно требовать однородности ортогональных составляющих или первых производных и т. д. В каждом конкретном случае в $S^{q}$ возникает замкнутое пространство, при этом допускаемый диапазон индекса гладкости $q$ будет зависеть от типа краевых условий.

\section{Л И ТЕ Р А Т У Р А}

1. Pыbым Р. Изв. АН ЭССР, Физ. Матем., 32, № 3, 246-256 (1983).

2. Рыъым Р. Изв. АН ЭССР, Физ. Матем., 32, № 1, 19-28 (1983).

3. Ладыженская О. А. Зап. науч. семинаров ЛОМИ АН СССР, 27, 1972, 91-115.

4. Ладыженская О. А. Зап. науч. семинаров ЛОМИ АН СССР, 115, 1982, 137-155.

5. Бабин А. В., Вицини М. Н. Зап. науч. семинаров ЛОМИ АН СССР, 115, 1982, $3-15$.

6. Градитейн И. С., Рыжик И. М. Таблицы интегралов, сумм, рядов и произведений. М., Физматгиз, 1962.

7. Функциональный анализ (под общей редакцией С. Г. Крейна). М., «Наука», 1972.

8. Ладыженская O. А. Математические вопросы динамики вязкой несжимаемой жидкости. М., «Наука», 1970.

9. Ладыюенская O. А. Смешанная задача для гиперболического уравнения. М., Гостехиздат, 1953. 


\section{LAPLACE'I OPERAATORI OMAVAR̃RTUSPROBLEEMI LAHEND RISTKULIKUS MAÁRATUD SOLENOIDAALSETE, OSAL PIIRKONTUURIL HOMOGEENSETE FUNKTSIOONIDE RUUMIS}

On vaadeldud ülesannet (1), (2) töös [ $\left.{ }^{1}\right]$ konstrueeritud Hilberti ruumis $U^{1}$. Probleemi lahendamiseks on kasutatud ortogonaalesitust solenoidaalsete perioodiliste funktsioonide

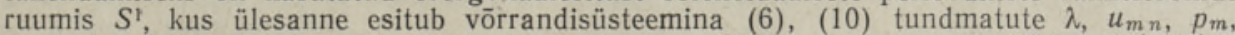
$q_{m}$ suhtes. Lahend on leitav funktsiooniridadena (27), kusjuures omaväärtused leitakse vōrrandeist (22) ja (25). Nende ridade summeerimine annab omafunktsioonide süsteemi (29). On uuritud selle süsteemi omadusi; muu hulgas on näidatud, et (29) moodustavad fundamentaalsüsteemi ruumides $U^{q}$, kui $q<3 / 2$, ning ortogonaalse baasi ruumides $U^{\prime}$ ja $U^{0}(33)$. Lisaks on ehitatud ortogonaalne baas $(35)$ ortogonaaltäiendis $W^{0}=S^{0} \backslash U^{0}$.

R. $R O O M$

\section{THE SOLUTION OF THE EIGENVALUE PROBLEM OF THE LAPLACE OPERATOR IN THE SPACE OF FUNCTIONS, SOLENOIDAL IN A RECTANGLE AND HOMOGENEOUS ON ONE PART OF THE BOUNDARY}

This paper is a continuation of [1]. Equations (1) are discussed in the case of boundary conditions (2). The problem is transformed into a variational equation (3) in the space $U^{1}\left(\left[{ }^{1}\right], 15,33\right)$. Equations $(5)-(7)$ are obtained by using the basis $\left(\left[{ }^{1}\right], 10\right)$. Variation of the equations (5) and (7) according to $\bar{v}_{m n}$ yields equations (6) and (10) for the finding of the unknowns $u_{m n}, p_{m}, q_{m}$ and $\lambda$. The complete system of linearly independent solutions is represented by formulae (12)-(14), where $S_{m}(\lambda)$ and $T_{m}(\lambda)$ are characteristic functions. The zeroes $S_{m}(\lambda)$ and $T_{m}(\lambda)$ determine the spectrum of eigenvalues, which can be obtained from- $(20)-(26)$. In coordinate representation eigenfunctions have the form of a functional series (27). Summation of these series yields (29). Eigenfunctions (29) are solenoidal, infinitely differentiable, and turn to zero if $x^{2}=0, x^{2}=1$. They satisfy equations (31). It is proved that the system (29) is complete in spaces $U^{q}$ if $q<3 / 2$ and orthogonal in $U^{0}$ and $U^{1}$. The supplementation of the system (29) by the functions (35) yields a system which is complete in space $S^{0}\left(\left[^{1}\right], 10\right)$. The functions $(35)$ are solenoidal vectors that can be represented as gradients (see (36)), and thus these functions are harmonic. 